\title{
L'INDICE FOLIAIRE DES COUVERTS FORESTIERS : MESURE, VARIABILITÉ ET RÔLE FONCTIONNEL
}

\author{
Nathalie BRÉDA
}

Dans un contexte climatique et stationnel donné, l'un des facteurs majeurs de la production d'un écosystème est son indice foliaire (Leaf Area Index pour les anglophones, abrégé par LAI), c'est-à-dire sa surface de feuilles exprimée par unité de surface au sol. La relation entre ce paramètre et la productivité est évidente: l'indice foliaire représente la surface par laquelle les flux de carbone (échanges photosynthétiques) et d'eau (transpiration) transitent.

L'accumulation de matière sèche dépend ainsi de l'indice foliaire, du rayonnement incident, et de l'efficience de conversion de ce rayonnement via la photosynthèse en matière sèche. Les agronomes connaissent bien ce fonctionnement et sont désormais capables de prédire le rendement d'une culture à partir de son indice foliaire. Cette démarche est d'ailleurs utilisée couramment comme moyen de contrôle et de taxation des rendements à large échelle, les indices foliaires étant estimés par télédétection. Toutefois, pour nos principales espèces forestières, les gammes de fluctuation de ce paramètre du couvert et le déterminisme de ses variations sont encore mal connus, tandis que les liaisons productivité / indice foliaire à l'échelle du peuplement commencent seulement à être prospectées.

\section{QU'EST-CE QUE L'INDICE FOLIAIRE ET COMMENT SE MESURE-T-IL ?}

L'indice foliaire est donc une grandeur sans dimension, qui exprime la surface foliaire d'un arbre, d'un peuplement, d'un écosystème ou d'un biome (1) par unité de surface de sol. Selon l'échelle à laquelle on s'intéresse, l'unité de surface de sol peut être le $\mathrm{m}^{2}$, l'hectare, le km². Pour un arbre, la surface de référence retenue est la surface de projection horizontale de son houppier. Les méthodes de détermination de l'indice foliaire peuvent être classées en deux catégories: les méthodes utilisant une mesure directe de surface foliaire et les méthodes optiques ou indirectes.

(1) Biome : unité écologique de très vaste étendue, recouvrant une fraction d'un ou plusieurs continents et caractérisée par un ensemble d'espèces végétales et animales qui lui sont spécifiques (exemples: toundra boréale, savane tropicale, forêt feuillue tempérée...). 
Pour les espèces à feuilles caduques, la plus simple des méthodes directes consiste à collecter les retombées de litières à l'automne sur des superficies échantillons connues et d'en mesurer ou évaluer la surface. Ainsi, les feuilles collectées peuvent être séchées et le poids de feuilles sèches converti en surface à partir d'un coefficient appelé surface spécifique (surface de feuille par unité de poids sec). Ce coefficient s'établit en mesurant la surface foliaire d'échantillons de litières, prélevés à différentes dates au cours de la chute, à l'aide d'un planimètre. Les échantillons sont ensuite séchés à l'étuve et la surface spécifique est calculée. Pour les essences à feuilles persistantes, la collecte des litières ne donne accès qu'à la fraction renouvelée chaque année de l'indice foliaire. II faut avoir recours à l'abattage et à la mesure de la surface foliaire d'arbres de statuts et de diamètres divers. Des tarifs sont établis (relations allométriques entre surface foliaire et diamètre ou, mieux, section conductrice) et permettent d'évaluer l'indice foliaire du peuplement à partir d'un histogramme de distribution des diamètres des tiges. Cette méthode est destructive et lourde à mettre correctement en œuvre.

Le couvert des peuplements forestiers intercepte le rayonnement solaire incident essentiellement au niveau des feuilles (accessoirement au niveau du tronc et des branches) : cette propriété a été utilisée depuis longtemps pour apprécier indirectement l'indice foliaire depuis le sol. En effet, à partir de modèles d'extinction du rayonnement incident par le couvert, une simple mesure d'éclairement simultanément en dessous et à l'extérieur du couvert (éclairement relatif) permet de calculer l'indice foliaire par inversion de la loi d'extinction du rayonnement de Beer-Lambert (Monsi et Saeki, 1953). Une autre méthode utilisée depuis longtemps consiste à prendre une photographie bien contrastée de la voûte du peuplement, en utilisant un objectif "fish-eye" (permettant une visée sur $360^{\circ}$ ) pour estimer la fraction de trouées dans le couvert (Ducrey, 1975). Le dépouillement de tels clichés, appelés photographies hémisphériques, est long et délicat, car il nécessite un seuillage manuel des gris avant d'estimer la proportion de surfaces noires (feuillage) par rapport aux surfaces blanches (ciel). Cette technique pourrait être réactualisée grâce aux progrès de l'analyse d'image et à l'avènement des appareils photographiques numériques, qui devraient permettre d'automatiser l'exploitation des images.

Commercialisés depuis quelques années, des appareils de mesure optique spécifiques, appelés analyseurs de couvert, permettent de réaliser des estimations d'indice foliaire non destructives, rapides et précises (Chason et al., 1991; Dufrêne et Bréda, 1995). Le principe de mesure d'interception d'un rayonnement (direct ou diffus) est repris par ces capteurs, et des modèles de calculs plus précis de la probabilité de contact d'un rayon lumineux avec un élément de feuillage, tenant compte en outre de l'inclinaison des feuilles et de l'angle d'incidence du rayonnement, ont été développés pour calculer l'indice foliaire (Campbell, 1986 ; Lang, 1986 ; Norman et Campbell, 1989).

Enfin, la télédétection (aéroportée ou satellitale) ouvre une autre voie possible de quantification indirecte de l'indice foliaire, à plus grande échelle (grandes parcelles, massifs forestiers, région ou continent). II ne s'agit plus de quantifier le rayonnement transmis sous couvert, mais d'évaluer au-dessus du couvert la réflectance du feuillage, dans des gammes de longueurs d'ondes spécifiques (domaine optique) en la distinguant de la réflectance propre du sol. De nombreux modèles physiques ou semi-empiriques de transferts radiatifs se développent et divers indices de végétation sont définis (Guérif et al., 1997). Un des plus anciens de ces indices de végétation est le NDVI (Normalized Difference Vegetation Index, Asrar et al., 1984) ; il utilise la différence normalisée entre les réflectances dans l'infrarouge et dans le rouge. Toutefois, la relation entre ces indices de végétation et l'indice foliaire n'est sensible que dans les faibles valeurs d'indice foliaire (inférieur à 4), ce qui limite leur utilisation pour les couverts forestiers. Dans des gammes de fort indice foliaire, la sensibilité d'autres indices de végétation est actuellement prospectée. 


\section{LES FACTEURS DE VARIATION DE L'INDICE FOLIAIRE}

L'indice foliaire d'un peuplement forestier dépend de sa composition en espèces, de la structure du couvert souvent liée à son traitement sylvicole (densité, degré de fermeture, stratification verticale...), de la physiologie des espèces et de l'état sanitaire des arbres. Indépendamment de ces caractéristiques de structure et composition du peuplement, ce paramètre est contrôlé par les facteurs climatiques et les conditions stationnelles, en particulier l'alimentation hydrique et la disponibilité en éléments minéraux dans le sol. Selon ces différents paramètres et conditions, l'indice foliaire des peuplements forestiers en zone tempérée peut varier entre 1 et 20 . II est généralement plus faible dans les peuplements feuillus (de 3 à 6) que dans les peuplements résineux (pouvant atteindre 20) (Waring et Schlesinger, 1985). Quelques espèces feuillues à croissance rapide (Eucalyptus, Peupliers par exemple) peuvent atteindre des indices extrêmes de 16 à 45 en culture intensive (Isebrands et Michael, 1986). En forêt équatoriale, l'indice foliaire ne dépasserait pas une valeur moyenne 8 (Alexandre, 1981). En fait, la limite maximale que l'indice foliaire peut atteindre est telle que les feuilles les plus basses dans le couvert reçoivent un peu plus que le rayonnement minimum vital (Alexandre, 1981). Ce maximum dépend de la capacité des feuilles à survivre à l'ombre, de l'architecture du couvert, en particulier de l'inclinaison des feuilles, de leur agrégation, et de leur distribution verticale. C'est ainsi que les forts indices foliaires de certains peuplements résineux sont possibles grâce aux effets d'agrégation des aiguilles qui permettent une pénétration de la lumière profondément dans le couvert.

L'âge et la densité (d'ailleurs généralement liés) sont souvent mentionnés comme facteurs de variation de l'indice foliaire, mais peu de relations ont été publiées sur la durée d'une révolution. Lorsqu'un peuplement forestier croît, son indice foliaire augmente jusqu'à un maximum, puis se stabilise ou diminue légèrement. En fait, ni l'âge ni la densité ne sont sans doute les paramètres explicatifs directs, et l'indice foliaire maximum serait plutôt atteint lorsque le couvert se ferme. Baldocchi et Hutchison (1986) considèrent que la fermeture du couvert des forêts commence pour un LAI supérieur à 3. L'âge de fermeture du couvert dépend quant à lui de l'espèce, du type de régénération, de la sylviculture appliquée... Ainsi, la relation entre densité et indice foliaire varie selon les espèces : chez un Sapin (Abies lasiocarpa), l'indice foliaire augmente avec la densité, alors que chez un Pin (Pinus contorta), il reste constant dans une large gamme de densités (Jack et Long, 1991). Les auteurs interprètent ces différences comme conséquences de la tolérance à l'ombre du Sapin. L'indice maximum est atteint à des âges différents selon les espèces (Schulze, 1982) : 16 ans chez des peuplements d'Épicéa de Sitka en Angleterre et de Pin en Suède, 32 ans chez le Peuplier tremble dans le Wisconsin, 50 ans pour le Sapin au Japon. Souvent, l'indice foliaire des peuplements de conifères augmente jusque vers 40 ans, puis il atteint une valeur d'équilibre qui ne change plus ensuite.

Pour une espèce donnée et dans un même massif forestier, le type de peuplement modifie également l'indice foliaire total en structurant la répartition verticale du feuillage et en introduisant des espèces d'accompagnement. Ainsi, en forêt domaniale de la Harth (2) située dans la plaine d'Alsace, la présence de sous-étage de Charme et de Tilleul en taillis-sous-futaie de chênes conduit à des indices foliaires totaux plus élevés qu'en futaie (figure 1, p. 139). De même, le mélange avec une espèce résineuse conduit à des indices foliaires plus forts. À noter que les différences entre taillis-sous-futaie pauvre (TSF-), moyen (TSFo) ou riche (TSF+), définies sur le terrain par rapport à un critère de surface terrière en Chêne (essence de l'étage dominant), ne se traduisent pas par des différences significatives d'indice foliaire : ceci reflète la forte contribution des essences du sous-étage (ici Charme et Tilleul) à l'indice foliaire total.

(2) Ces données ont été acquises dans le cadre d'une convention de recherche entre I'INRA-Nancy, l'Office national des Forêts et l'Observatoire écologique de la Harth (ONF, Division de Mulhouse). 
Dans des peuplements à couvert clair (situation typique en pinède), le faible indice foliaire de l'étage dominant $(2,5$ à 3,5$)$ laisse pénétrer une quantité importante de rayonnement au niveau du sol, ce qui favorise le développement d'une strate herbacée dont l'indice foliaire est significatif. Ainsi, en plantation de Pin maritime dont l'indice foliaire est égal à 3,0, Berbigier et al. (1991) estiment l'indice foliaire de la strate herbacée composée de Molinie à 1,2, soit presque $30 \%$ de l'indice foliaire total. Une synthèse de Satoo (1970) indique que la contribution du sous-étage à l'indice foliaire total en forêt décidue est très variable (de 15 à $60 \%$ de LAl total) mais souvent significative, l'indice foliaire total variant peu autour de 7.

L'indice foliaire des peuplements varie aussi au cours de l'année : cette dynamique saisonnière est évidente pour les espèces à feuilles caduques où le peuplement passe d'un stade hivernal sans feuille à une phase progressivement feuillée jusqu'à atteindre un maximum vers le milieu de saison (figure 2, p. 139). Ici, à partir de mesures de rayonnement relatif, on peut détecter dès le débourrement l'expansion rapide de la première vague de croissance, ainsi qu'une augmentation de presque 1 point d'indice foliaire consécutive à une seconde vague de croissance entre mi-juin et fin juin. La valeur maximale du LAl est atteinte vers la mi-août. Mais des variations intra-annuelles peuvent aussi être observées en peuplement résineux [de 3,2 à 5,3 chez Pinus strobus (Vose et Swank, 1990), ou chez Pinus radiata (Whitehead et al., 1994)], en forêt tropicale ou sempervirente. Raison et al. (1992) indiquent des variations intraannuelles de LAl jusqu'à 3 selon les conditions de milieu chez Pinus radiata. Ces variations en cours d'année reflètent un décalage entre la mise en place des aiguilles ou des feuilles de l'année (typiquement en début de saison de végétation) et la chute des aiguilles ou feuilles âgées (maximum en fin d'été ou automne). Peu d'études ont suivi les dynamiques temporelles de production et de perte d'aiguilles (Aussenac, 1969, 1979) et surtout évalué leurs conséquences en terme de variation de LAl (Raison et al., 1992). Une des raisons majeures est que les relations allométriques classiquement utilisées pour estimer les LAI résineux ne permettent pas de quantifier ces variations intra-annuelles. Des informations partielles pourront être obtenues sur les placettes résineuses du réseau RENECOFOR ${ }^{(3)}$, mais les données ne pourront être interprétées qu'en biomasse et non en variation d'indice foliaire. Dans tous les cas, il convient donc de préciser à quelle période correspond l'indice foliaire mesuré. Classiquement, la comparaison d'indices foliaires entre peuplements est réalisée sur la valeur maximale observée au cours de la saison.

Une autre dynamique temporelle s'ajoute aux cinétiques intra-annuelles décrites ci-dessus : il s'agit de modifications d'une année sur l'autre ou sur plusieurs années de la valeur maximale de l'indice foliaire (Bréda et Granier, 1996). Ces variations interannuelles peuvent être observées après des années très sèches. Elles atteignent 2 points d'indice foliaire dans le cas d'une chênaie en Lorraine (figure 3, p. 139) et sont réversibles: il ne faut alors pas interpréter la réduction de surface de feuilles comme un mécanisme de dépérissement, mais plutôt comme un mode de régulation à moyen terme. Enfin, des accidents climatiques (gel de printemps) ou biotiques (défoliateurs) peuvent avoir des conséquences directes l'année de l'accident (diminution du nombre de feuilles) et différées : la mise en place d'un nouveau cycle de feuilles et la réduction d'indice foliaire après l'accident affectent la quantité de réserves carbonées disponibles pour la mise en place d'une feuillaison normale lors de la saison suivante. Outre ces modifications naturelles, les interventions sylvicoles, qu'elles soient coupes de taillis ou éclaircies, réduisent l'indice foliaire du peuplement. Selon l'intensité et la nature (systématique ou non, dans l'étage dominant ou dans le sous-étage) de l'intervention, cette réduction est ou non proportionnelle au nombre de tiges ôtées ; immédiatement après éclaircie, elle est

(3) Le Réseau national de suivi à long terme des Écosystèmes forestiers (RENECOFOR) a été mis en place à partir de 1991 par l'Office national des Forêts pour fournir une base objective permettant d'interpréter une variation quelconque de la santé des forêts. II s'agit d'un suivi intensif et continu sur du long terme (30 ans minimum), réalisé sur 102 placettes représentatives des 10 essences principales de la forêt française. 
Figure 1

INFLUENCE DU TYPE DE PEUPLEMENT

FFP : futaie feuillue pure

TS : taillis simple

TSF- : taillis-sous-futaie pauvre

TSFo : taillis-sous-futaie moyen

TSF+ : taillis-sous-futaie riche

TSF-FSS : intermédiaire entre

taillis-sous-futaie et futaie sur souche

FSS : futaie sur souche

FRM : futaie résineuse mélangée

(Convention ONF-INRA,

Observatoire écologique de la Harth) Chaque point est une moyenne de $\mathrm{n}$ placettes,

n étant indiqué à côté de chaque point. L'écart-type est représenté SUR L'INDICE FOLIAIRE DE 62 CHÊNAIES EN FORÊT DOMANIALE DE LA HARTH

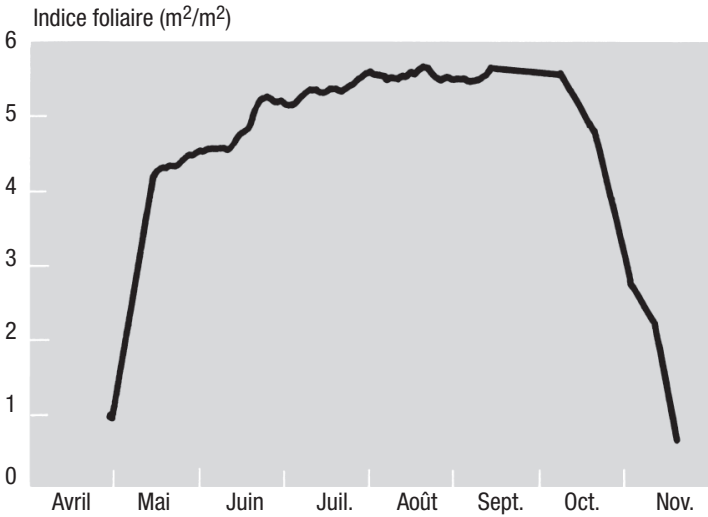

Indice foliaire $\left(\mathrm{m}^{2} / \mathrm{m}^{2}\right)$

EXEMPLE DE VARIATION SAISONNIÈRE D'INDICE FOLIAIRE DANS UNE CHÊNAIE, calculé à partir de mesures d'interception du rayonnement global par le couvert pendant la saison de végétation et par ramassage des litières à l'automne (forêt domaniale de Champenoux, Meurthe-et-Moselle)

Figure 3

EXEMPLE DE VARIATION INTERANNUELLE D'INDICE FOLIAIRE

estimé par ramassage de litières dans un peuplement de chênes de 30-40 ans (forêt domaniale de Champenoux, Meurthe-et-Moselle)

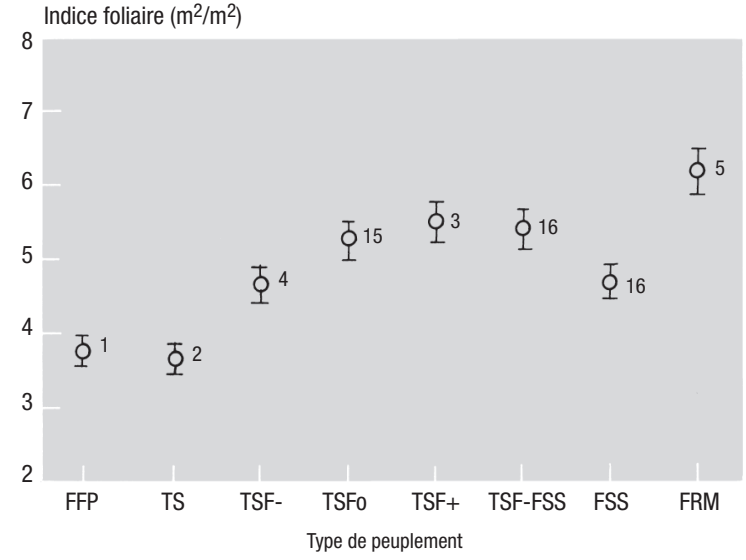


davantage proportionnelle à la réduction de surface terrière qu'au nombre de tiges, à condition que l'intervention concerne l'étage dominant. Ainsi, dans une plantation de Douglas âgée de 19 ans, une éclaircie systématique prélevant une ligne sur deux (50\% du nombre de tiges et de la surface terrière) a conduit à une réduction de $43 \%$ de l'indice foliaire estimé à partir de mesures d'éclairement relatif et en supposant que le coefficient d'extinction est le même dans les deux traitements (LAI témoin = 9,2, LAl éclairci = 5,2; Aussenac et al., 1982). Une éclaircie par le haut dans un perchis de chênes supprimant $20 \%$ du nombre de tiges et $35 \%$ de la surface terrière a provoqué une réduction de $35 \%$ de l'indice foliaire (Bréda et al., 1995). Dans un taillis de Chêne vert, Ducrey et Huc (1998) observent une réduction proportionnelle de ces deux paramètres, qui se conserve plusieurs années après l'intervention. Inversement, Cutini (1996) montre une réduction plus forte (63 à $83 \%$ ) de la surface de feuilles que de la surface terrière (50-60\%), lors d'éclaircies de conversion de taillis de Chêne chevelu en futaie sur souche. Après cette diminution brutale au cours de la première saison de végétation suivant l'éclaircie, le couvert se referme progressivement et l'indice foliaire augmente d'année en année, plus rapidement que la surface terrière : la relation entre ces deux variables n'est alors plus conservée. Malheureusement, très peu de mesures interannuelles sont actuellement disponibles pour quantifier la dynamique de l'indice foliaire au cours de cette phase de retour à un couvert fermé. II semble que 4 à 6 saisons de végétation soient souvent suffisantes pour revenir aux valeurs précédant l'éclaircie. Cette dynamique dépend de l'essence, de l'âge du peuplement et des conditions stationnelles, et peut être largement modulée par les conditions climatiques des années consécutives à l'intervention.

L'hétérogénéité spatiale au sein d'une même parcelle forestière peut aussi être considérable. Typiquement, à l'échelle d'une parcelle, l'indice foliaire varie du simple au double, en raison d'une trouée, d'un bouquet plus dense, d'un changement dans le sous-étage. Cette hétérogénéité spatiale a par exemple été très bien mise en évidence par Walter et Himmler (1995) dans divers peuplements de Pin sylvestre d'âges différents. Estimé à partir de photographies hémisphériques, l'indice foliaire varie fortement dans la parcelle la plus jeune (rangs de plantation, LAl entre 2,0 et 7,8 ) et moins (entre 2,3 et 5,4 ) dans les parcelles d'âge moyen. Dans un taillis de Chêne vert, selon Ducrey et Huc (1998), il varie entre 2,1 et 4,9 selon la densité du couvert sur des parcelles de $900 \mathrm{~m}^{2}$. Même sur de courtes distances, une forte variabilité intra-peuplement peut être mise en évidence par des mesures rapprochées à l'aide d'un analyseur de couvert depuis le sol ou de collecteurs de litières. Ainsi, Joffre et al. (1996) mesurent des indices foliaires compris entre 2,3 et 3,6 sur un placeau de $30 \mathrm{~m}^{2}$ de taillis de Chêne vert. De même, la figure 4 (p. 141) illustre les variations détectées le long d'un cheminement linéaire de $250 \mathrm{~m}$ d'orientation aléatoire, en forêt alluviale (Erstein, Haut-Rhin). Dans cette réserve hors gestion depuis de nombreuses années, la chênaie-frênaie alluviale suit une dynamique naturelle avec apparition de trouées lors de la chute d'arbres morts. Les mesures ont été réalisées tous les 2,50 m avec un analyseur de couvert (Li-Cor, LAl-2000) ; I'indice foliaire fluctue selon la distance par rapport aux trouées ou aux arbres dominants dont le houppier est très large, et selon la densité du feuillage qui varie entre espèces. Cette forte variabilité implique un grand nombre de répétitions de mesures (collecteurs de litières, photographies hémisphériques ou mesures portatives) pour déterminer l'indice foliaire d'une parcelle forestière. En l'absence de telles répétitions échantillonnant toute la surface de la parcelle, l'indice foliaire mesuré ne peut avoir qu'une signification locale, alors précisément appréhendée par 3 ou 4 répétitions de mesures depuis le sol (Wang et al., 1992).

La forte influence de la disponibilité en eau du sol sur l'indice foliaire est bien établie par les biogéographes à très large échelle, couvrant des biomes très contrastés (steppes, prairies, forêts sempervirentes, feuillues ou résineuses) : l'indice foliaire des grands types de végétation décroît avec la disponibilité en eau (Gholz et al., 1990). À l'échelle des forêts conifères du continent nord-américain, Grier et Running (1977), Waring et al. (1978) et Gholz (1982) ont montré clairement qu'il était corrélé avec les précipitations. Plus précisément, il serait contrôlé 
par le déficit climatique, c'est-à-dire la différence entre l'évapotranspiration potentielle et les précipitations (Grier et Running, 1977 ; Gholz, 1982 ; figure 5, ci-dessous). Toutefois, à l'échelle d'une région climatiquement homogène, les liaisons entre indice foliaire et disponibilité en eau restent à établir. Une étude de Landsberg (1986) montre que les variations interannuelles de longueur d'aiguilles de Pinus radiata sont corrélées à la pluviométrie de la saison de croissance. Même sur les sites pauvres et non fertilisés qu'il étudiait, l'irrigation permettait dans tous les cas d'augmenter l'indice foliaire. Dans nos travaux réalisés dans l'Est de la France et en Allemagne, l'indice foliaire (mesuré au maximum saisonnier) de peuplements équiennes et monospécifiques, à couvert fermé, feuillus (Chênes et Hêtres) et résineux (Douglas, Épicéas), apparaît d'autant plus élevé que la réserve utile du sol est forte (figure 6, ci-dessous). Nous reviendrons plus loin sur l'interprétation en terme de bilan hydrique de cette relation entre la potentialité hydrique du sol et l'indice foliaire.

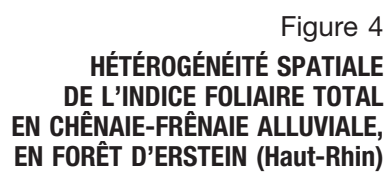

Chaque point correspond à une mesure ponctuelle espacée de 2,50 m le long d'un cheminement de $250 \mathrm{~m}$ (analyseur de couvert Li-Cor, LAl-2000)

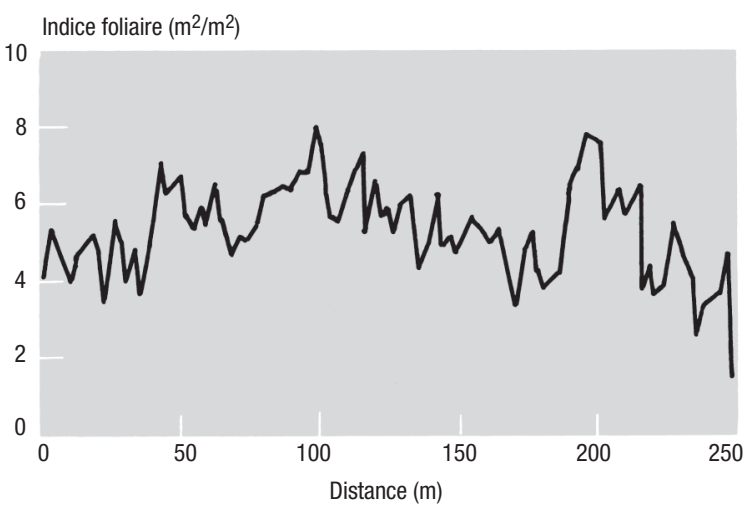

Figure 5

RELATION ENTRE INDICE FOLIAIRE DÉVELOPPÉ (qui prend en compte la forme des aiguilles) ET DÉFICIT CLIMATIQUE AU COURS DE LA SAISON DE VÉGÉTATION (pluie-ETP) pour différents types de végétation rencontrés le long d'un transect ouest-est en Oregon (d'après Gholz, 1982)

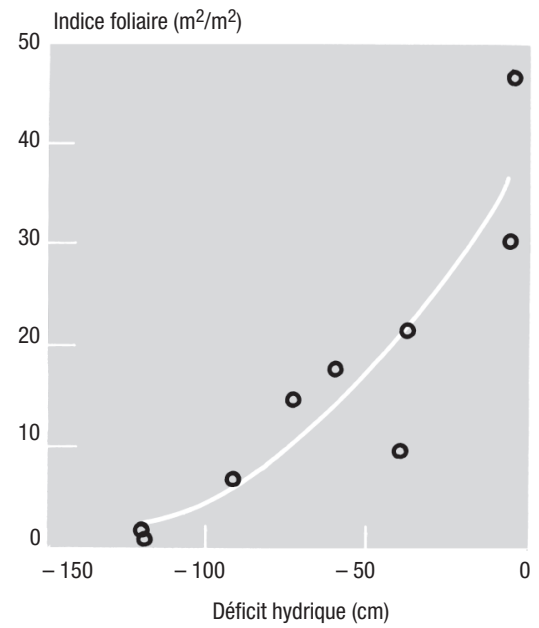

Figure 6

RELATION ENTRE INDICE FOLIAIRE MAXIMAL ET RÉSERVE UTILE DU SOL

dans des peuplements équiennes et monospécifiques feuillus et résineux de l'Est de la France et en Allemagne

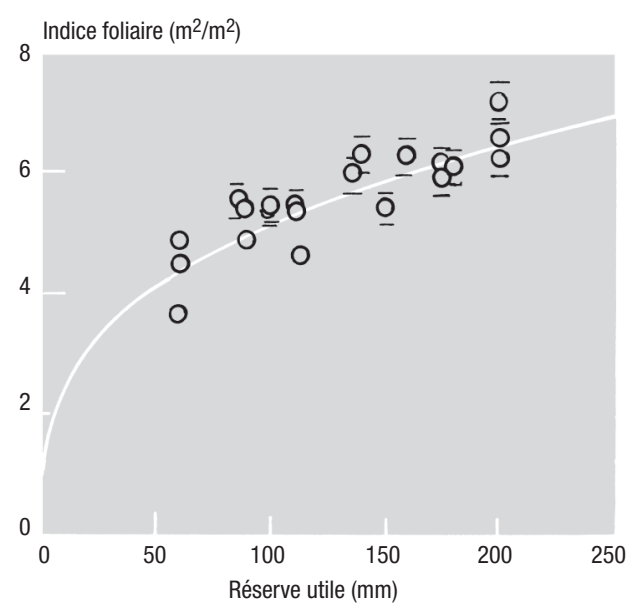


Enfin, la teneur en éléments minéraux du sol semble également susceptible de limiter le développement de l'indice foliaire sur les stations pauvres. Ainsi, une fertilisation azotée a permis d'augmenter l'indice foliaire de peuplements de Pinus taeda de plus de $60 \%$ par rapport aux peuplements non traités sur des sites très déficients, alors que l'apport de phosphore était sans effet sur l'indice foliaire (Vose et Allen, 1988). Dans des peuplements de 10 ans de Pinus radiata installés sur sols pauvres sur granite, un traitement par irrigation et fertilisation a permis d'augmenter le LAl de 2,5 à 3,5 en une saison de végétation. En deux ans, le LAl a augmenté de 2,5 à 3,9-4,4 dans les traitements irrigués ou fertilisés, tandis qu'il a atteint 6 ou 6,5 dans les traitements irrigués et fertilisés (Landsberg, 1986). De tels résultats ne sont pas disponibles actuellement pour des essences forestières feuillues.

\section{RÔLE FONCTIONNEL DE L'INDICE FOLIAIRE DANS LE BILAN EN EAU DES PEUPLEMENTS FORESTIERS}

Les différents flux du bilan de l'eau en forêt (transpiration des arbres, évaporation du sol et transpiration de la strate herbacée, interception des précipitations, écoulement, drainage...) dépendent étroitement des caractéristiques du couvert forestier (Aussenac et al., 1995). L'indice foliaire, tant par sa valeur que par sa variation au cours de l'année, est la variable fonctionnelle de structure du couvert qui gouverne ces différents flux. C'est aussi un des paramètres explicatifs essentiel des différences de fonctionnement hydrique entre couvert à feuilles caduques et couvert résineux (LAl plus élevés et persistance toute l'année).

\section{LAl et interception des pluies}

La quantité de pluie atteignant le sol, paramètre essentiel à la recharge de la réserve en eau du sol (flux entrant), dépend avant tout de l'intensité de la pluie incidente (Aussenac, 1968), mais décroît lorsque l'indice foliaire augmente. En région tempérée, l'interception annuelle représente 15 à $25 \%$ de la pluie incidente en peuplement feuillu et 30 à $50 \%$ en peuplement résineux (Aussenac, 1968), soit 15 à $25 \%$ de plus chez les conifères. Cette différence s'explique par des indices foliaires généralement plus élevés en peuplement résineux, mais aussi par la présence d'une période défeuillée chez les feuillus pendant laquelle les précipitations sont importantes et l'interception est faible. Ces différences d'interception entre feuillus et résineux conduisent à un bilan hydrique et un drainage vers les nappes qui peuvent être de $20 \%$ plus forts en peuplement feuillu qu'en peuplement résineux (Stanhill, 1970, in : Schulze, 1982).

Cependant, le degré de fermeture du couvert modifie la relation entre indice foliaire et interception des précipitations (Aussenac, 1970 ; Aussenac et Boulangeat, 1980). Ainsi, pour un même indice foliaire, des peuplements de chênes éclaircis interceptent davantage de pluie que des peuplements à couverts fermés, en raison à la fois d'une plus grande capacité de saturation des couronnes plus isolées et à une évaporation plus rapide de l'eau interceptée grâce aux turbulences créées par l'irrégularité du couvert (figure 7, p. 143). Les conséquences de la discontinuité de couvert se retrouvent également chez les pins, qui présentent des interceptions relativement fortes pour leurs faibles indices foliaires (interception variant entre 14 à $22 \%$ de la pluie incidente pour une pinède d'indice foliaire de 3,0 ; Loustau et al., 1992).

\section{LAl et transpiration du peuplement et du sous-bois}

La transpiration d'un couvert forestier est déterminée par les conditions d'évapotranspiration potentielle (demande climatique) et la disponibilité en eau dans le sol. Lorsque cette disponibilité n'est pas limitante et qu'il n'y a pas de régulation par les stomates, la transpiration est maximale et proportionnelle à la surface foliaire cumulée. Il est alors possible de prédire la transpiration maximale du peuplement à partir de son indice foliaire et des conditions d'éva- 
potranspiration (Granier et Bréda, 1996) (figure 8, ci-dessous). Ainsi, la transpiration maximale d'un peuplement de Chêne rapportée à l'évapotranspiration potentielle varie d'une année sur l'autre proportionnellement à l'indice foliaire entre 3 et 6 .

Figure 7

RELATION ENTRE INDICE FOLIAIRE ET INTERCEPTION DES PRÉCIPITATIONS

exprimée en pourcentage de la pluie incidente (moyenne sur la période feuillée) dans des peuplements de Chênes à couvert fermé (ronds noirs) ou à couvert ouvert (éclaircie et coupes d'ensemencement, triangles ouverts)

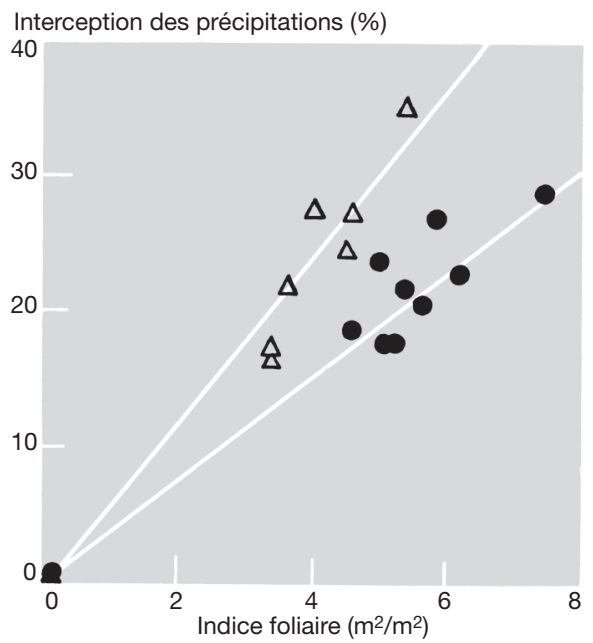

Figure de synthèse d'après Carlisle et al., 1965 ; Aussenac, 1975 ; Nizinski et Saugier, 1989 ; Bréda, 1994 et Bréda données non publiées
Figure 8

RELATION ENTRE LA TRANSPIRATION MAXIMALE (T)

mesurée par flux de sève en absence de contrainte hydrique rapportée à l'évapotranspiration potentielle (ETP, formule de Penman) ET L'INDICE FOLIAIRE (ronds : témoin ; triangles noirs : éclairci)

T/ETP

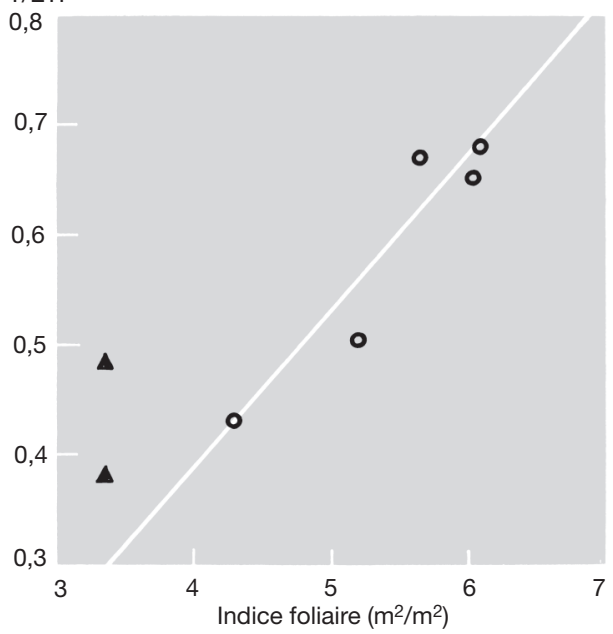

D’après Bréda et Granier, 1996.

Toujours en condition de bonne disponibilité en eau dans le sol, l'évaporation du sol et la transpiration du sous-bois sont généralement proportionnelles à la quantité de rayonnement net qui pénètre jusqu'à la surface du sol. Cette fraction de rayonnement non intercepté dépend directement de l'indice foliaire et peut être calculée comme une fonction inverse de l'indice foliaire. L'évaporation du sol ne représente que $5 \%$ de l'évaporation totale lorsque l'indice foliaire atteint 4 (Denmead, 1969) alors que cette proportion peut atteindre $50 \%$ si l'indice foliaire chute en dessous de 2. Cela signifie que l'évapotranspiration de l'écosystème se répartit entre la transpiration des arbres, d'autant plus forte que l'indice foliaire est élevé, et l'évaporation du sol et du sous-bois, inversement proportionnelle à l'indice foliaire des arbres. La répartition des flux d'évapotranspiration peut atteindre de 70 à $80 \%$ pour les couverts forestiers à faible indice foliaire et 20 à $30 \%$ pour le sous-bois (Roberts et al., 1980 ; Granier et al., 1990 ; Loustau et Cochard, 1991). En peuplement feuillu, la contribution du sous-bois évolue donc en cours de saison, inversement à la dynamique de l'indice foliaire de l'étage dominant : au printemps, alors que le sol est bien alimenté en eau et que les arbres n'ont pas encore toutes leurs feuilles, la contribution du sous-bois est majoritaire ; plus tard, lorsque l'indice foliaire du couvert atteint sa valeur maximale, le rayonnement au sol et la disponibilité en eau à la surface du sol sont réduits et la transpiration des arbres devient prépondérante. De fait, on peut dire que, même si la contribution respective de chaque strate varie, la consommation globale en eau de l'écosystème (arbres + sous-bois) reste pilotée par l'indice foliaire global du peuplement (Whitehead et al., 1994). 


\section{LAl et indice de stress hydrique}

Les modulations de ces divers flux d'eau par l'indice foliaire interagissent et conditionnent la quantité d'eau disponible dans le sol. Ainsi, la réserve utile du sol diminuera plus vite et plus fort au cours de la saison si l'indice foliaire du couvert est plus élevé (figure 9, ci-dessous) : la transpiration et l'interception sont plus fortes, mais l'évaporation du sol et du sous-étage reste faible. Ainsi, la date à laquelle la réserve en eau du sol atteint le seuil de déficit hydrique qui affecte le fonctionnement des arbres est plus précoce, et l'épuisement de la réserve sera plus intense. Les conséquences d'une modification d'indice foliaire sur la disponibilité en eau du sol et sur les risques de déficit hydrique sont difficiles à prédire et à généraliser, en particulier parce qu'elles dépendent des caractéristiques climatiques de chaque année. Selon la répartition des précipitations au cours de l'année et les conditions d'évapotranspiration potentielle, une modification d'indice foliaire affectera plutôt l'interception ou la transpiration.

Figure 9

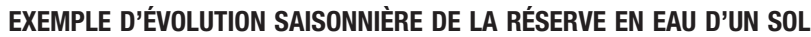

de $100 \mathrm{~mm}$ de réserve utile simulée pour quatre peuplements de chênes d'indices foliaires respectifs de 3,5, 4,5, 5,5 et 7

(données climatiques journalières Météo-France, poste de Bâle, Modèle Granier et Bréda)

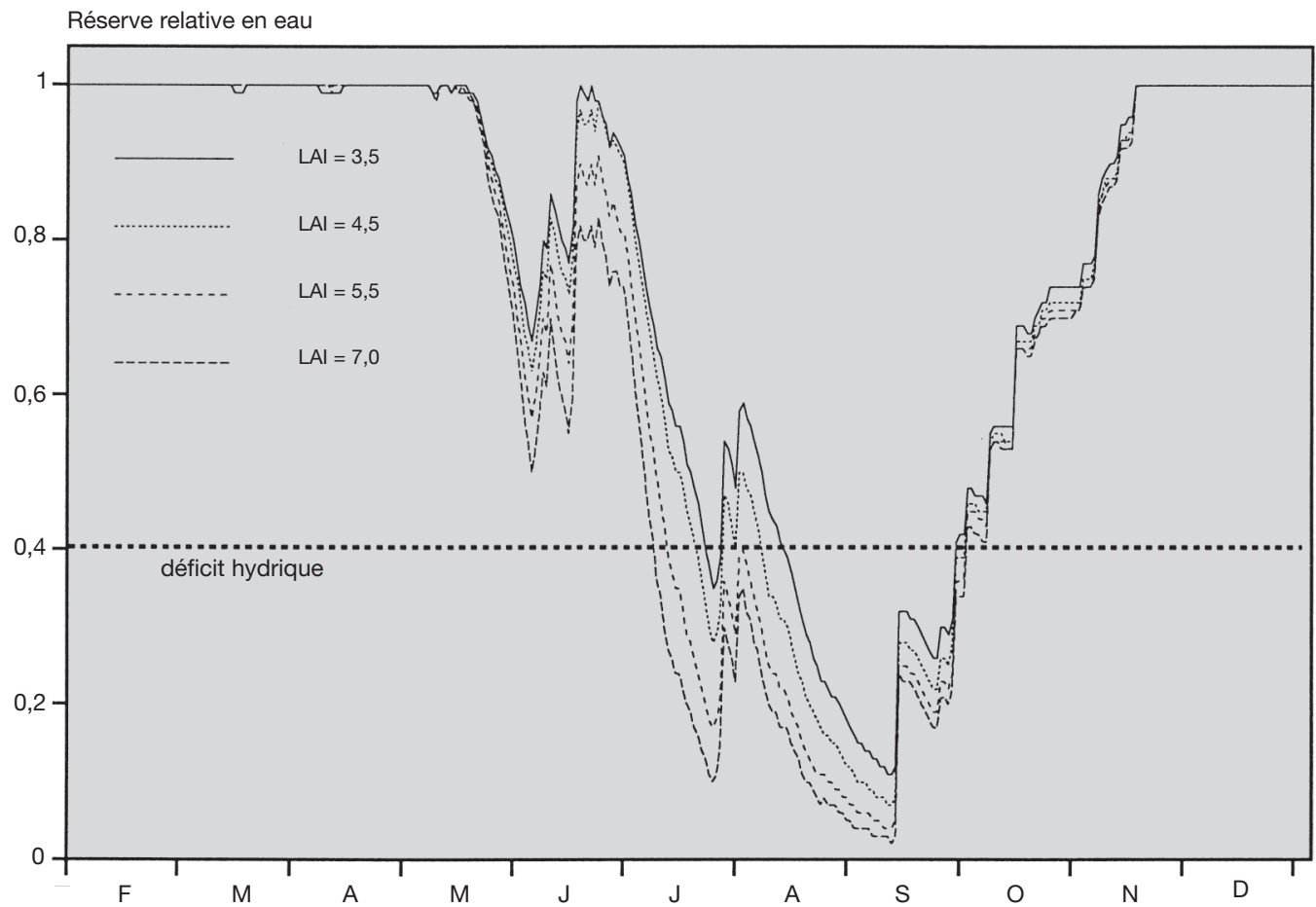

L'utilisation d'un modèle de bilan hydrique journalier dans lequel les flux d'eau sont pilotés par l'indice foliaire du peuplement (Granier et al., 1995) peut aider à comparer la sensibilité du déficit hydrique à des variations d'indice foliaire pour des années à profil pluviométrique contrasté. On constate ainsi que l'augmentation du déficit hydrique n'est pas proportionnelle à la variation d'indice foliaire et que cette différence varie selon les années (figure 10, p. 145). 
Figure 10

INCIDENCE DE L'INDICE FOLIAIRE SUR L'INDICE

DE STRESS (durée et intensité du déficit hydrique) simulés pour 5 années climatiquement contrastées (données climatiques journalières Météo-France, poste de Bâle, Modèle Granier et Bréda)

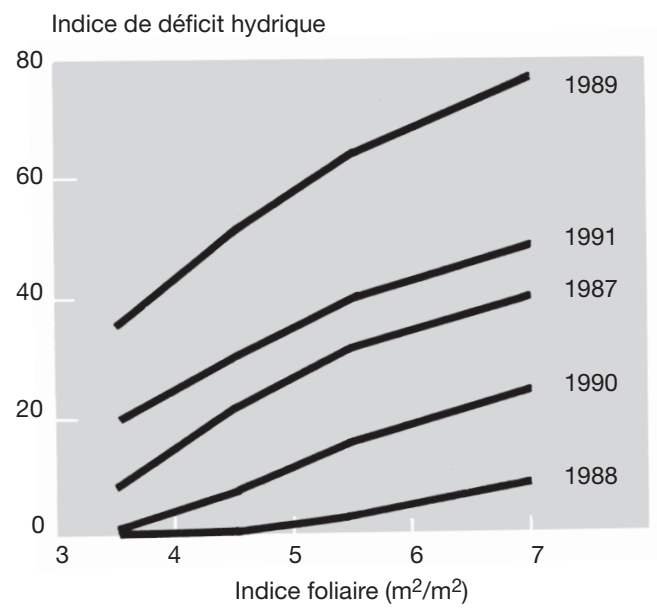

Figure 11

RELATION ENTRE PRODUCTIVITÉ PRIMAIRE NETTE DE LA PARTIE AÉRIENNE ET INDICE FOLIAIRE DÉVELOPPÉ

(qui prend en compte la forme des aiguilles) pour différents types de végétation rencontrés le long d'un transect ouest-est en Oregon (d'après Gholz, 1982)

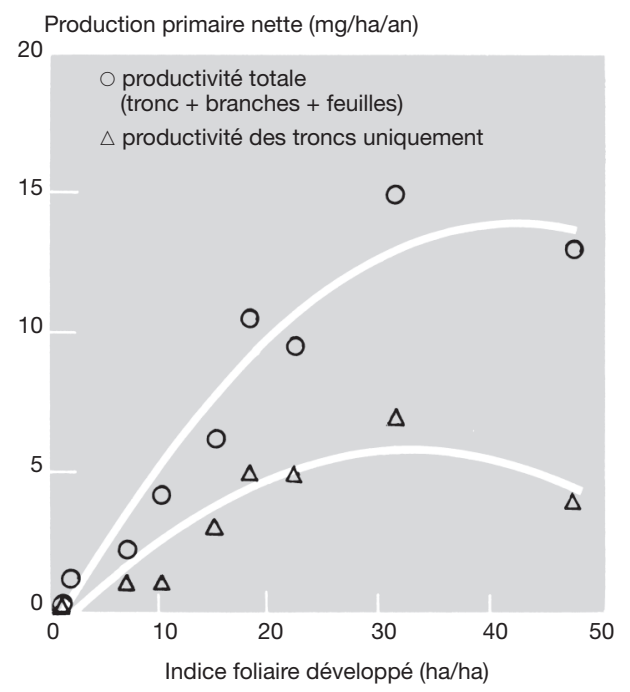

\section{INDICE FOLIAIRE ET PRODUCTIVITÉ PRIMAIRE NETTE}

La productivité primaire nette (en abréviation anglo-saxonne NPP) des peuplements forestiers est couramment corrélée positivement avec l'indice foliaire jusqu'à un seuil, au-delà duquel la productivité diminue très souvent, essentiellement en raison de la mortalité et des effets d'inhibition de l'efficience photosynthétique des feuilles de la base du couvert par la compétition. II peut également y avoir interaction avec un bilan hydrique qui devient défavorable lorsque le LAl augmente (figure 11, ci-dessus, d'après Gholz, 1982 ; Gholz et al., 1990). L'existence d'un LAI optimum a été largement débattue. Au-delà de cet optimum (appelé parfois LAI critique), une augmentation du LAl et de la fixation de $\mathrm{CO}_{2}$ ne peut plus contrebalancer la réduction de l'assimilation des surfaces foliaires déjà en place en raison de l'ombrage créé par les nouvelles feuilles. Par exemple, la productivité primaire nette d'un peuplement de Douglas est maximale pour environ la moitié de l'indice foliaire maximal et diminue pour des indices plus forts, parce que la perte de croissance par mortalité des arbres dépasse le faible accroissement des arbres restants (Waring, 1983). Dans les peuplements de Pinus elliotii Engelman, en Floride, la productivité primaire nette annuelle augmente rapidement pendant les 10 à 15 premières années puis diminue ensuite rapidement. Elle reste étroitement corrélée au LAI jusque tard dans la révolution. Après 18 ans, elle décline alors que l'accumulation de biomasse se ralentit, tandis que la surface de feuilles est maintenue. Après l'âge normal de rotation (environ 30 ans), les deux variables diminuent alors que la structure du peuplement se stabilise à un niveau de productivité moindre (Gholz, 1986). Dans les jeunes peuplements, l'importance du LAI pour la croissance est modifiée par la longueur de la période d'activité photosynthétique. Ainsi, la productivité des peuplements résineux est favorisée par rapport aux feuillus. Kira (1975) a montré une forte corrélation entre la productivité nette à la fois de forêts déci- 
dues et sempervirentes et la durée du LAI (définie comme le produit du LAl et de la longueur de la saison de croissance en mois). Ces études se limitent à des régions spécifiques montrant une corrélation positive entre productivité et LAI, mais de larges variations peuvent être observées et sont alors associées à des différences entre photosynthèse nette, respiration des tissus vivants, ou encore la quantité de carbone allouée aux racines (Gholz, 1986). Waring (1983) souligne que la corrélation entre productivité et indice foliaire n'est pas maintenue dans les régions où la sécheresse et les sols pauvres prédominent.

Figure 12 ÉVOLUTION INTERANNUELLE DE LA TRANSPIRATION ANNUELLE, DE L'INTERCEPTION DES PRÉCIPITATIONS, DU DÉFICIT HYDRIQUE ET DE L’ACCROISSEMENT RADIAL ANNUEL DES CERNES

dans deux peuplements voisins de Hêtre et d'Épicéa du Kaiserstuhl (Allemagne)

(réserve utile du sol : $190 \mathrm{~mm}$, indice foliaire du Hêtre : 6,2, indice foliaire de l'Épicéa : 8,0). Données climatiques journalières Météo-France, poste de Bâle (Projet européen $n^{\circ}$ EV-5V-CT94-0437)

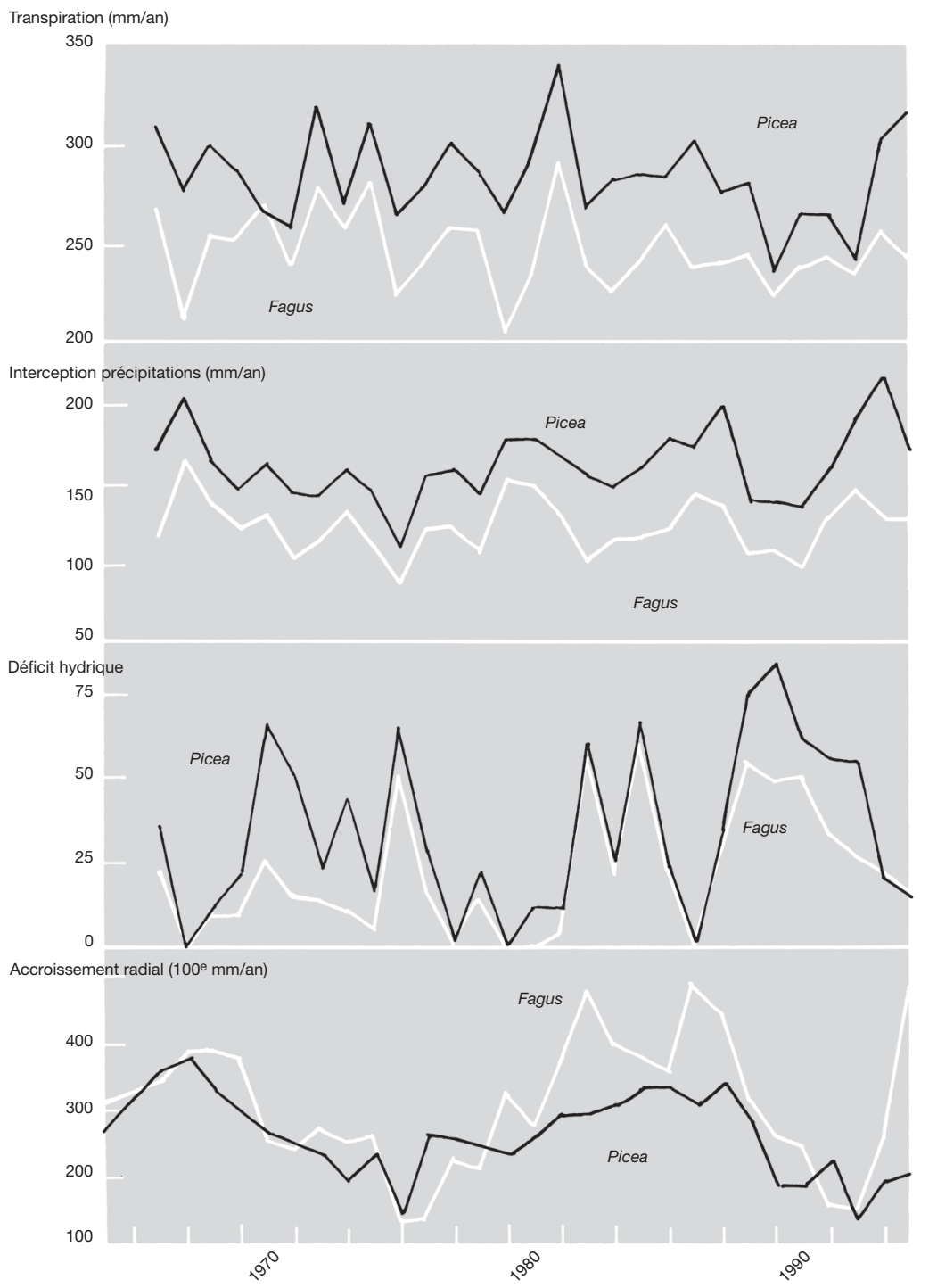




\section{INDICE FOLIAIRE ET CROISSANCE : COMPARAISON FEUILLUS ET RÉSINEUX}

En déterminant à la fois la transpiration du peuplement, le rayonnement disponible au niveau du sol et l'interception des précipitations, la surface de feuilles mesurée par l'indice foliaire joue donc un rôle clé dans le fonctionnement hydrique et carboné des couverts, et gouverne également la productivité. La figure 12 (p. 146) illustre les variations interannuelles sur une série de 30 ans des flux d'eau essentiels (transpiration et interception) qui ont été simulées pour une hêtraie et une pessière installées côte à côte sur un sol limoneux profond en Allemagne ${ }^{(4)}$. Les différences d'indice foliaire du couvert et de longueur de saison feuillée entre Épicéa et Hêtre conduisent à une transpiration et une interception des précipitations plus élevées en pessière. Toutefois, les différences entre les deux peuplements ne sont pas constantes d'une année sur l'autre, en raison de l'interaction entre structure du couvert et répartition des pluies. Le déficit en eau du sol résultant de ces différents flux d'entrée et de sortie d'eau de l'écosystème montre également de forts écarts entre les deux types de couverts avec, selon les années, des déficits plus forts sous pessière ou comparables dans les deux peuplements. Dans ces conditions, la croissance radiale des hêtres est souvent meilleure que celle des épicéas, mais elle est aussi plus fluctuante d'une année sur l'autre. La chute de croissance est souvent plus forte l'année consécutive à un déficit hydrique fort.

En conclusion, l'indice foliaire est une bonne expression des échanges d'énergie et de matière entre le sol, les couverts forestiers et l'atmosphère. Par son action physique, il détermine le microclimat en dessous du couvert ainsi que la quantité de pluie interceptée ou qui atteint le sol, conditionnant les flux du sous-étage. II représente par ailleurs la partie fonctionnelle du couvert, siège des échanges gazeux (transpiration et photosynthèse) produisant les assimilats nécessaires à la croissance et aussi au stockage de réserves indispensables pour la mise en place du feuillage de la saison suivante. De plus, l'indice foliaire est un paramètre dynamique de la structure du couvert, subissant à la fois des variations saisonnières et interannuelles importantes. Si ses variations interannuelles commencent à être admises, leur quantification, leur déterminisme et leurs conséquences en terme de bilan de carbone et de croissance sont encore mal connus et évalués. L'indice foliaire conditionne à la fois les flux de matière et d'énergie des couverts forestiers, mais il dépend en retour du fonctionnement écophysiologique des arbres et des peuplements: cette interdépendance rend délicate l'étude de ses variations. Plusieurs programmes de recherches s'attachent à répondre à ces questions essentielles pour la compréhension des bilans hydrique, carboné et minéraux des écosystèmes forestiers et de leur productivité.

\section{Remerciements}

L'auteur remercie F. Willm, B. Clerc, F. Geremia, J.-M. Walter et Marianne Peiffer, pour leur contribution à la collecte fastidieuse de certaines données rassemblées ici (litières, précipitations, mesures de LAI).

(4) Ces résultats ont été obtenus dans le cadre du projet européen n EV-5V-CT94-0437 intitulé "Dendroecological analysis of climate-growth relations of five important European tree species along an East-West transect : Black Forest - Vosges Mountains - Lorraine Plain" et coordonné par le Professeur Spiecker (Université de Fribourg). 


\section{Nathalie BRÉDA}

\section{BIBLIOGRAPHIE}

ALEXANDRE (D.Y.). - L'Indice foliaire des forêts tropicales. - Acta Oecologica, 4, 1981, pp. $299-312$.

ASRAR (G.), FUCHS (M.), KANEMASU (E.T.), HATFIELD (J.L.). - Estimating absorbed photosynthetic radiation and leaf area index from spectral reflectance in wheat. - Agron. Journal, 76, 1984, pp. 300-306.

AUSSENAC (G.). - Action du couvert forestier sur la distribution au sol des précipitations. - Annales des Sciences forestières, vol. 27, 1970, pp. 383-399.

AUSSENAC (G.). - Couverts forestiers et facteurs du climat: leurs interactions, conséquences écophysiologiques chez quelques résineux. - Nancy: Université Nancy I, 1975. - 234 p. (Thèse Doctorat ès Sciences).

AUSSENAC (G.). - Interception des précipitations par le couvert forestier. - Annales des Sciences forestières, vol. 25, 1968, pp. 135-156.

AUSSENAC (G.). - Production de litière dans divers peuplements forestiers de l'Est de la France. - Oecologia Plantarum, vol. IV, 1969, pp. 225-236.

AUSSENAC (G.). - Production de litière dans quatre jeunes peuplements de Douglas de l'Est de la France. Revue forestière française, vol. XXXI, $\mathrm{n}^{\circ} 1,1979$, pp. 15-19.

AUSSENAC (G.), BOULANGEAT (C.). - Interception des précipitations et évapotranspiration réelle dans des peuplements de feuillu (Fagus silvatica L.) et de résineux [Pseudotsuga menziesii (Mirb.) Franco]. - Annales des Sciences forestières, vol. 37, 1980, pp. 91-107.

AUSSENAC (G.), GRANIER (A.), BRÉDA (N.). - Effets des modifications de la structure du couvert forestier sur le bilan hydrique, l'état hydrique des arbres et la croissance. - Revue forestière française, vol. XLVII, $n^{\circ} 1$, 1995, pp. 54-62.

AUSSENAC (G.), GRANIER (A.), NAUD (R.). - Influence de l'éclaircie sur la croissance et le bilan hydrique d'un jeune peuplement de Douglas [Pseudotsuga menziesii (Mirb.) Franco]. - Canadian Journal of Forest Research, 12, 1982, pp. 222-231.

BALDOCCHI (D.D.), HUTCHISON (B.A.). - On estimating canopy photosynthesis and stomatal conductance in a deciduous forest with clumped foliage. - Tree Physiology, 2, 1986, pp. 155-168.

BERBIGIER (P.), DIAWARA (A.), LOUSTAU (D.). - Étude microclimatique de l'effet de la sécheresse sur l'évaporation d'une plantation de pins maritimes et du sous-bois. - Annales des Sciences forestières, vol. 48, 1991, pp. 157-177.

BRÉDA (N.). - Analyse du fonctionnement hydrique des Chênes sessile (Quercus petraea) et pédonculé (Q. robur) en conditions naturelles; effets des facteurs du milieu et de l'éclaircie. - Nancy : Université Henri-Poincaré, 1994. - 59 p. + publications (Thèse de Doctorat).

BRÉDA (N.), GRANIER (A.). - Intra- and inter-annual variations of transpiration, leaf area index and radial growth of a sessile oak stand (Quercus petraea). - Annales des Sciences forestières, vol. 53, 1996, pp. 521-536.

BRÉDA (N.), GRANIER (A.), AUSSENAC (G.). - Effects of thinning on soil water balance and trees water relations, transpiration and growth in an oak forest (Quercus petraea). - Tree Physiology, vol. 15, 1995, pp. 295306.

CAMPBELL (G.S.). - Extinction coefficients for radiation in plant canopies calculated using an ellipsoïdal inclination angle distribution. - Agric. For. Meteorol., vol. 36, 1986, pp. 317-321.

CARLISLE (A.), BROWN (A.H.F.), WHITE (E.J.). - The interception of precipitation by oak (Quercus petraea) on a high rainfall site. - Quarterly Journal of Forestry, April 1965, pp. 140-143.

CHASON (J.W.), BALDOCCHI (D.D.), HUTSON (M.A.). - A comparison of direct and indirect methods for estimating forest canopy leaf area. - Agric. For Meteorol., vol. 57, 1991, pp. 107-128.

CUTINI (A.). - The influence of drought and thinning on leaf area index estimates from canopy transmittance method. - Annales des Sciences forestières, vol. 53, 1996, pp. 595-603.

DENMEAD (O.T.). - Comparative micrometeorology of a wheat field and a forest of Pinus radiata. - Agric. For. Meteorol., 6, 1969, pp. 357-371.

DICKMANN (D.I.), STEINBECK (K.), SKINNER (T.). - Leaf area and biomass in mixed and pure plantations of Sycamore and Black Locust in the Georgia Piedmont. - Forest Science, vol. 31, 1985, pp. 509-517.

DUCREY (M.). - Utilisation des photographies hémisphériques pour le calcul de la perméabilité des couverts au rayonnement solaire. - Annales des Sciences forestières, vol. 32, 1975, pp. 73-92, 205-221.

DUCREY (M.), HUC (R.). - Effets de l'éclaircie sur la croissance et le fonctionnement écophysiologique d'un taillis de Chêne vert. - Revue forestière française, vol. LI, n² 2, 1999, pp. 326-340.

DUFRÊNE (E.), BRÉDA (N.). - Estimation of deciduous forest leaf area index using direct and indirect methods. - CEcologia, 104, 1995, pp. 156-162.

GHOLZ (H.L.). - Environmental limits on aboveground net primary production, leaf area, and biomass in vegetation zones of the pacific northwest. - Ecology, 63, 1982, pp. 469-481.

GHOLZ (H.L.), EWEL (K.C.), TESKEY (R.O.). - Water and forest productivity. - Forest Ecology and Management, vol. 30, 1990, pp. 1-18.

GRANIER (A.), BADEAU (V.), BRÉDA (N.). - Modélisation du bilan hydrique des peuplements forestiers. - Revue forestière française, vol. XLVII, $\mathrm{n}^{\circ}$ spécial "Modélisation de la croissance des arbres forestiers et de la qualité des bois", 1995, pp. 59-68. 
GRANIER (A.), BOBAY (V.), GASH (J.H.C.), GELPE (J.), SAUGIER (B.), SHUTTLEWORTH (W.J.). - Vapour flux density and transpiration rate comparisons in a stand of Maritime Pine (Pinus pinaster Ait.). In : Les Landes forest. - Agric. For. Meteorol., vol. 51, 1990, pp. 309-319.

GRANIER (A.), BRÉDA (N.). - Modelling canopy conductance and stand transpiration of an oak forest from sap flow measurements. - Annales des Sciences forestières, vol. 53, 1996, pp. 537-546.

GRIER (C.C.), RUNNING (S.W.). - Leaf area of mature northwestern coniferous forests : relation to site water balance. - Ecology, vol. 58, 1977, pp. 893-899.

GUÉRIF (M.), LAGOUARDE (J.-P.), NICOLAS (H.). - Variables de fonctionnement des couverts végétaux issues des données de télédétection dans les domaines optiques et infrarouge thermique. In: Actes de l'ÉcoleChercheurs, INRA en Bioclimatologie, Le Croisic, 25-29 mars 1996. Tome 2 : du couvert végétal à la région. - Département de bioclimatologie - INRA Éd., 1997. - pp. 81-108.

ISEBRANDS (J.G.), MICHAEL (D.A.). - Effects of leaf morphology and orientation on solar radiation interception and photosynthesis in Populus. In : Crown and Canopy structure in relation to productivity / T. Fujimori et D. Whitehead Eds. - Ibaraki (Japon) : Forestry and Forest Products Research Institute, 1986. - pp. 359-381.

JACK (S.B.), LONG (J.N.). - Response of leaf area index to density for two contrasting tree species. - Canadian Journal of Forest Research, vol. 21, 1991, pp. 1760-1764.

JOFFRE (R.), RAMBAL (S.), ROMANE (F.). - Local variations of ecosystem functions in Mediterranean evergreen oak woodland. - Annales des Sciences forestières, vol. 53, 1996, pp. 561-570.

KIRA (T.). - Primary production of forests. In: Photosynthesis and productivity in different environments / J.P. Cooper Ed. - Cambridge University Press, 1975. - pp. 5-40.

LANDSBERG (J.J.). - Experimental approaches to the study of the effects of nutrients and water on carbon assimilation by trees. - Tree Physiology, 2, 1986, pp. 427-444.

LANG (A.R.G.). - Leaf area and average leaf angle from transmittance of direct sunlight. - Australian Journal of Botany, vol. 34, 1986, pp. 349-355.

LOUSTAU (D.), BERBIGIER (P.), GRANIER (A.), EL HADI MOUSSA (F.). - Interception loss, throughfall and stemflow in a maritime pine stand. I- Variability of throughfall and stemflow beneath the pine canopy. - Journal of Hydrology, 138, 1992, pp. 449-467.

LOUSTAU (D.), COCHARD (H.). - Utilisation d'une chambre de transpiration portable pour l'estimation de l'évapotranspiration d'un sous-bois de Pin maritime à Molinie [Molinia coerulea (L.) Moench]. - Annales des Sciences forestières, vol. 48, 1991, pp. 29-45.

MAASS (J.M.), VOSE (J.M.), SWANK (W.T.), MARTINEZ-YRIZAR (A.). - Seasonal changes of leaf area index (LAI) in a tropical deciduous forest in west Mexico. - Forest Ecology and Management, vol. 74, 1995, pp. 171180.

MONSI (M.), SAEKI (T. ). - Über den Lichtfactor in den Pflanzengesellschaften und seine Bedeutung für die Stoffproduction. - Jpn. J. Bot., vol. 14, 1953, pp. 22-52.

NIZINSKI (J.J.), SAUGIER (B.). - A model of transpiration and soil-water balance for a mature oak forest. Agric. For. Meteorol., vol. 47, 1989, pp. 1-17.

NORMAN (J.M.), CAMPBELL (G.S.). - Canopy structure. In : Plant physiological ecology : field methods and instrumentation / R.W. Pearcy, J. Ehlringer, H.A. Mooney, P.W. Rundel Eds. - London and New York : Chapman and Hall, 1989. - pp. 301-325.

RAISON (R.J.), KHANNA (P.K.), BENSON (M.L.), MYERS (B.J.), MCMURTRIE (R.E.), LANG (A.R.G.). - Dynamics of Pinus radiata foliage in relation to water and nitrogen stress. Il- Needle loss and temporal changes in total foliage mass. - Forest Ecology and Management, vol. 52, 1992, pp. 159-178.

RAISON (R.J.), MYERS (B.J.), BENSON (M.L.). - Dynamics of Pinus radiata foliage in relation to water and nitrogen stress. I- Needle production and properties. - Forest Ecology and Management, vol. 52, 1992, pp. 139158.

ROBERTS (J.), PYMAR (C.F.), WALLACE (J.S.), PITMAN (R.M.). - Seasonal changes in leaf area, stomatal and canopy conductances and transpiration from bracken below a forest canopy. - Journal of Applied Ecology, vol. 17, 1980, pp. 409-422.

SATOO (T.). - A synthesis of studies by the harvest method : primary production relations in the temperate deciduous forest of Japan. In: Analysis of temperate forest ecosystems / D.E. Reichle Ed. - Springer-Verlag, 1970.

SCHULZE (E.D.). - Plant life forms and their carbon, water and nutrient relations. In: Physiological plant ecology II. - Encyclopedia of Plant Physiology, New series, vol. 12B., 1982, pp. 616-667.

VOSE (J.M.), ALLEN (H.L.). - Leaf area, stemwood growth, and nutrition relationships in Loblolly Pine. - Forest Science, vol. 34, 1988, pp. 547-563.

VOSE (J.M.), SWANK (W.T.). - Assessing seasonal leaf area dynamics and vertical leaf area distribution in eastern white pine (Pinus strobus L.) with a portable light meter. - Tree Physiology, vol. 7, 1990, pp. 125134.

WALTER (J.M.), HIMMLER (C.G.). - Spatial heterogeneity of a Scots pine canopy : an assessment by hemispherical photographs. - Canadian Journal of Forest Research, vol. 26, 1995, pp. 1610-1619. 
WANG (Y.S.), MILLER (D.R.), WELLES (J.M.), HEISLER (G.M.). - Spatial variability of canopy foliage in an oak forest estimated with fisheye sensors. - Forest Science, vol. 38, 1992, pp. 854-865.

WARING (R.H.). - Estimating forest growth and efficiency in relation to canopy leaf area. - Adv. Ecol. Res., vol. 13, 1983, pp. 327-354.

WARING (R.H.), EMMINGHAM (W.H.), GHOLZ (H.L.), GRIER (C.C.). - Variation in maximum leaf area of coniferous forests in Oregon and its ecological significance. - Forest Science, vol. 24, 1978, pp. 131-140.

WARING (R.H.), SCHLESINGER (W.H.). - Forest Ecosystems : concepts and management. - New York: Academic Press, 1985. - 340 p.

WHITEHEAD (D.), KELLIHER (F.M.), FRAMPTON (C.M.), GODFREY (M.J.S.). - Seasonal development of leaf area in a young, widely spaced Pinus radiata D. Don stand. - Tree Physiology, vol. 14, 1994, pp. 1019-1038.

WHITEHEAD (D.), KELLIHER (F.M.), LANE (P.M.), POLLOCK (D.S.). - Seasonal partitioning of evaporation between trees and understorey in a widely spaced Pinus radiata stand. - Journal of Applied Ecology, vol. 31, 1994, pp. 528-542.

\section{L'INDICE FOLIAIRE DES COUVERTS FORESTIERS : MESURE, VARIABILITÉ ET RÔLE FONCTIONNEL (Résumé)}

Après avoir rappelé la définition et les principales méthodes de détermination de l'indice foliaire des peuplements forestiers, les facteurs de variations de ce paramètre sont décrits. L'âge, la densité, la composition spécifique, le type de peuplement ou encore les potentialités du milieu (disponibilité en eau et en éléments nutritifs) influencent l'indice foliaire. Ses variations temporelles, qu'elles soient saisonnières ou entre années, sont décrites. Le rôle fonctionnel de l'indice foliaire dans les bilans hydrique, carboné et minéral des peuplements est rappelé et illustré sur les principaux flux d'eau de l'écosystème. L'interception des précipitations et la transpiration du peuplement principal aussi bien que du sous-bois sont pilotées par l'indice foliaire, qui ainsi gouverne une part importante de la dynamique et de l'intensité du dessèchement du sol sous le couvert forestier. II intervient enfin de manière complexe sur la croissance des arbres et la productivité des écosystèmes.

\section{THE LEAF AREA INDEX OF FOREST CANOPIES : MEASUREMENT, VARIABILITY AND FUNCTIONAL ROLE (Abstract)}

Beginning with the definition of the leaf area index and of the major methods for determining it in forest stands, the article then describes the variation factors for this parameter. Age, density, specific composition and type of stand as well as the potential of the environment (water and nutrient availability) influence the leaf area index. Variations over time, whether seasonal or from one year to the next, are also discussed. The functional role of the leaf area index in respect of the water, carbon and mineral balances of stands is recalled and illustrated for the principal water flows in the ecosystem. Interception of rainfall and transpiration by the overstory as well as the undergrowth are dependent on the leaf area index, which is hence a major determinant in the dynamics and intensity of soil drainage beneath the forest canopy. Finally, it also has a complex effect on tree growth and ecosystem productivity. 